\title{
ROLE OF MRI IN EVALUATION OF ANTERIOR CRUCIATE
}

\section{LIGAMENT TEAR}

\author{
Sajjad Hussain (Corresponding author) \\ Student, Superior College Lahore, University Campus, 17-KM Raiwaind Road, Kot Arain, Lahore, Pakistan
}

Mob: +923419474287 E-mail: hsajjad867@gmail.com

Suman Mehdi

Student, Superior College Lahore, University Campus, 17-KM Raiwaind Road, Kot Arain, Lahore, Pakistan

Mob: +923085458085 E-mail: sumanmehdi108@gmail.com

Utrooba shahid

Lecturer, Superior College Lahore, University Campus, 17-KM Raiwaind Road, Kot Arain, Lahore, Pakistan

Mob: +923074798892 E-mail: atroobashahid@gmail.com

Hafiz Muhammad Rizwan

MRI technologist, Lahore general hospital

Lecturer, Superior College Lahore, University Campus, 17-KM Raiwaind Road, Kot Arain, Lahore, Pakistan

Mob: +923435135072 E-mail: dr.rzwaan@gmail.com

Rana Muhammad Athar Azeem Shams

Lecturer, Superior College Lahore, University Campus, 17-KM Raiwaind Road, Kot Arain, Lahore, Pakistan

Mob: +92334-6996693 E-mail: atharshams44@gmail.com

Rana Muhammad Bakhtwar Khan Sajawal

Lecturer, Superior College Lahore, University Campus, 17-KM Raiwaind Road, Kot Arain, Lahore, Pakistan

Mob: +92304-2040204 E-mail: bksajawa177@gmail.com

Humaira Rauf

Assistant professor medical imaging technology, Fatima memorial hospital Lahore Pakistan.

E mail: humaira.rauf@fmhcmd.edu.pk

\begin{abstract}
Magnetic resonance imaging being a most advanced and non-invasive imaging modality is a gold standard imaging modality in the diagnosis of soft tissue injuries including the injuries of ligament and tendons because of its three dimensional approach. Almost there are 360 joints in human body with the help of which we can move from one place to other, among them knee joint is the largest joint. It is a synovial joint of hinge variety which mainly allows flexion and extension. Knee joint is supported by a huge network of ligaments and tendons which provide a great strength and flexibility to the knee joint. Anterior and posterior cruciate ligament are the important stabilizing ligaments of the knee joint these two ligaments connects the tibia with femur. Injury or torn to them can cause a critical clinical conditions to the patients suffering from that injury. Injury to anterior cruciate ligament is more frequent as compare to other ligamentous injuries of the knee. Injury to ACL occurs mostly by sudden change in direction so because of which it affects the people who take part in different types of
\end{abstract}


sports like basketball, soccer, baseball skiing. Female athletes are at higher risk of having ACL tear as compare to males. ACL tear should be treated properly because injury to it can cause a serious clinical conditions like it can cause the immobilization of the subject until it is treated properly. For the accurate treatment there should be most advanced and highly sensitive diagnostic approach which aids in the addition of accurate information regarding the location, grading and the condition of ACL tear either there is complete or partial tear. Magnetic resonance imaging modality can diagnosis ACL tears with a high accuracy with the help of specific protocols which are more sensitive to soft tissue injuries.

Keywords: ANTERIOR CRUCIATE, LIGAMENT TEAR

DOI: $10.7176 / \mathrm{JHMN} / 93-10$

Publication date:September $30^{\text {th }} 2021$

\section{Introduction}

Magnetic resonance imaging is the fast, most advanced and non-invasive imaging technique that can produce three dimensional detailed images of anatomical structures of the body including various tendons and ligaments with high accuracy ${ }^{(1)}$. Almost there are 360 joints in human body with the help of which we are able to move. Joints play a vital role in the locomotion of any living being, joint or articulation is a place where two or more bones meet with the help of joints we can move from one place to other. Knee joint is the largest joint in the human body and the most frequently injured joint in the body ${ }^{(2)}$. It is a synovial joint of hinge Varity allowing us to sit, walk and jump. It is formed by articulation between proximal articular surface of tibia and distal articular surface of femur, in addition to it a third bone patella also takes part in the formation of knee joint.

Anterior cruciate ligament (ACL) is a crucial component of the knee joint because it stabilizes the knee joint and bears with the anterior and tibial movement and rotational loads. It is commonly injured structure occur in noncontact conditions during rapid change of direction. It is supported by vast network of muscles and ligaments, from the previous studies it was found that the injury (tear) to anterior cruciate ligament is most common and frequent as compare to other ligaments of the knee joint ${ }^{(3)}$. The injury of Anterior cruciate ligament (ACL) most commonly occur during sports in adolescents and young adults and also occurs in road traffic accidents that involves the sudden stop or changing in direction rapidly, in jumping movements and landing such in outdoor sports activities just like playing basketball, football and skiing and fast running. Mostly athletes are at higher risk of having ACL tear ${ }^{(4)}$.

From the previous researches it is found that in the region of United States the occurrence rate of ACL tear among the population is about 100000 to 200000 per year ${ }^{(5)}$. While similar study conducted in France showed the ACL injury rate is about 15000 cases per year ${ }^{(6)}$. From the given researches it was found that female athletes are more prone to receive ACL tear as compare to male participants ${ }^{(7)}$.

Female are 10 times greater risk from the ACL injury.it is because hormonal influence and anatomical variations in the body of females it is due to increased posterior tibial as well as meniscal slopes it is also due to decreased femoral notch sizes and smaller ligament sizes as compare to men. To determine these anatomical variations in the female body due to which they are more prone to have ACL tear further research is needed ${ }^{(8)}$.

Many people hear or feel a "pop" in the knee when subjected to ACL injury. Knee may become swollen, feel unstable and become intensely painful to bear weight. The anterior cruciate ligament (ACL), one of two ligaments that cross in the middle of the knee, connects femur to tibia and helps to stabilize the knee joint. Injury to ACL is a serious complication of the knee joint because the anterior cruciate ligament is the important stabilizer of knee joint and injury to it can be results in serious clinical conditions like it can cause the immobilization of the subject and does not recover when torned until it is treated accurately. For the accurate treatment there should be proper parameters to diagnose properly, there may be complete or partial tear. Common sign and symptoms of the ACL tear that the patients often describes is a loud pop with by severe pain and swelling in the knee joint. Severe pain and swelling makes walking and weight bearing very difficult and painful. If it ignored and remain untreated, the knee will feel unstable and the patient may complains of recurrent pain and swelling and giving way, specifically when walking on irregular ground surface or climbing up or down stairs.

Conventional radiographs and CTs are less sensitive in the diagnosis of acute ACL injury. Findings are indirect and confined to bone or Soft tissue pathologies and insufficient to diagnose the tear present in the anterior cruciate ligament. Plain X-rays are more sensitive specific for broken bones, other injuries that may mimic a torn ACL include fractures, in most cases patients suffering from an ACL tear, the X-ray findings are quite 
normal. To overcome this problem the radiologists suggest advanced modalities to gain proper accuracy for the diagnosis of anterior cruciate ligament tear. Magnetic resonance imaging being a most suitable imaging modality is a gold standard imaging modality in the diagnosis of soft tissue injuries including the injuries of ligament and tendon because of its three dimensional approach due to which Magnetic resonance (MR) imaging being a gold standard imaging modality of soft tissue injuries is a test of choice for early and accurate diagnosis of knee injuries especially for ligamentous injuries ${ }^{(9)}$.

MRI has particularly expanded the role of radiology in the assessment of acute knee injuries ${ }^{1}$. It is due to its high resolution ability to the soft tissues of the body and the capability of three dimensional imaging approach and specific protocols which are more sensitive to soft tissue injuries, in addition to precise diagnosis the injury it is also beneficial for the physicians and surgeons to decide the optimal treatment approaches. To achieve the higher sensitivity and specificity higher magnetic field strength is recommended so the magnetic strength of three tesla seems to be the better choice of modality to minimize the chances of uncertainties in the diagnosis of ligamentous injuries ${ }^{(10)}$. Commonly the anterior cruciate ligaments are evaluated on oblique sagittal MRIs with $\mathrm{T} 2$ weighted imaging technique. It is because of high sensitivity and specificity of T2 weighted imaging sequences towards the ACL tears as compare to T1 weighted imaging sequences.

For the characterization of the tear T1 weighted images acquired at least in one plane. Edema and effusion associated with ACL tear is more clearly visible on T2 weighted images ${ }^{(11)}$. In addition proton density imaging protocols are also used to assess the ligaments along with the fat saturation imaging protocols. Despite of this most of radiologists believe that multiplannar imaging technique, the addition of coronal and axial imaging planes are also important for correlation specifically when the suspected ACL is not clearly visible on sagittal images. The addition of axial and coronal plane images can add more important diagnostic information about ACL injury which lead to improve the diagnostic accuracy, confidence and precision of MRI regarding the severity of the ACL injury ${ }^{(12)}$.

Normally on both T1 and T2 weighted imaging sequences anterior cruciate ligament appears hypo intense straight bands parallel to intercondylar roof. Some high signal bright areas are also seen it is due to the presence of fat tissue or synovium. It fans out and descends to the anterior tibial attachment ${ }^{(13)}$.

To acquire a good quality of images the presence of highly experience medical imaging technologist is mandatory to minimize the risk of personal errors while performing the diagnostic test. Before performing this diagnostic procedure the magnetic imaging technologist should ensure that there should not be any electrical, magnetical or any other ferromagnetic surgical implant within the body of patient presented to MRI department for scanning purpose. By all these it makes MRI highly sensitive and accurate for the detection of ACL tear ${ }^{\text {s(14) }}$. The purpose of this study is to provide the systematic percentage of anterior cruciate ligament tear in South Asia in the territory of Pakistan based on advanced radiological techniques under the supervision of highly experienced and qualified radiologists and radiological technologists for the authentic results regarding the occurrence of anterior ligament tear among the population and to minimize the chances of uncertainty.

\subsection{Material and methodology}

This retrospective study was conducted in Lahore general hospital in radiology department under the supervision of highly trained and experienced magnetic resonance imaging technologists and radiologists including 60 adult patients.

Subjects suffering from severe pain and swelling in the knee joint which not relived by pain killer and antiinflammatory medications, with the previous history of road traffic accident or history of sport injury were included.

Claustrophobic patients and the patients with any type of electrical, magnetically or any other ferromagnetic surgical implants just like cardiac peace maker or the presence of any foreign body in eye or any other part of the body.

After ensuring that all the subjects are free from any magnetical, mechanical and any other type of ferromagnetic surgical implants. The patients suspected for ACL tear are placed in spine position one by one according to their respective turn. A specialized knee coil is raped around the painful and swollen knee for the diagnostic evaluation. The affected knee is assessed on different MRI protocols like T1 T2, PD FAT SAT. with the help of these protocols most of useful information regarding ACL tear was obtained in sagittal plane while for further 
more additional information other two planes coronal and axial plane are also considered. This study was performed on GE pioneer three tesla MRI scanner and with dedicated extremity coil. I e knee coil.

\subsubsection{Results}

In this study total 60 patients suffering from severe knee pain and swelling were included. 20 females $33.3 \%$ and 40 males patients $66.7 \%$. The youngest patient of this study was of 18 years and the oldest was of 62 years. Most of the patients in this study were between 18 to 40 years.

From the given study of 60 subjects the age group of 26 years are more affected accounting approximately $16.7 \%$. Patients of age group from 20 to 30 years are more affected from ACL tear.

Out of 60 patients 39 patients which accounts $65 \%$ were suffering from swelling with knee pain and 21 patients accounts about $35 \%$ were not suffering from knee swelling with pain.

All the subjects of given study were suffering from severe knee joint pain which it makes walking and weight bearing very difficult for the subjects.

From the diagnostic finding it was found that out of 60 patients presented to the radiology department with suspected ACL tear 45 patients which accounts about $75 \%$ were affected from the ACL tear while remaining 15 patients' accounts $25 \%$ were with unaffected ACL. Swelling and pain may be due some other reasons.

While performing this study it was found that out of 60 patients 28 patients accounts about $46.6 \%$ were suffering from complete ACL tear while 17 patients accounts $28.3 \%$ were suffering from partial ACL tear. Rest of 15 patients accounts $25 \%$ were remain unaffected.

\subsubsection{Discussion}

The purpose of this study is to rule out the incidence of ACL tear in the patients presented to a radiology department of Lahore general hospital with intense knee pain and swelling around the knee joint. The sample size of this study consists of 60 patients among which 20 were females and 40 patients were males. The age of the patients between 18 to 62 years.

After removing of the metallic as well as magnetic, electric and other ferromagnetic substances from the subject the patient is placed in supine position on the couch of the MRI scanner. A specialized coil is wrapped around the affected knee and assessed on specific MRI protocols designed specifically for the detection of ligamentous and soft tissue injuries. Standard protocols like T2WI PDW and FAT SAT are commonly used.

Avadesh p. sing et al., conducted a study under the topic of study of various knee injury findings in MRI. Total seventy five patients were added in their sample out of which 50 patients comprising $66.6 \%$ of the total number were male while 25 member comprising $33.3 \%$ were females.in this study male patients preponderance in distribution of knee injury was noted.

Out of total sample 5 patients approximately (6.67\%) were of age group from 1 to 20 years.42 members which accounts about (56\%) out of total number were of age group 21 to 40 years.26 patients which accounts about ( $34.7 \%$ ) were of age group 41 to 60 years. And the 2 patients which accounts about $(2.67 \%)$ were of 61 to 80 years. From the results it was found the young patients of age group between 21 to 40 years are most affected by the ACL tear while the age group of 61 to 80 years are least affected. Most of the knee injuries were caused by the road traffic accidents accounts approximately (40\%) and to some extent and geographical locations knee joint is commonly affected by sport injuries $(32 \%)$

\subsubsection{Conclusion}

Our study revealed that anterior cruciate ligament tear is a serious clinical condition which is mostly caused by road traffic accidents and also takes place in sports activities and has to be treated accurately before it get more worse. For proper cure of the injury there should be a precise and accurate diagnostic approaches which play a vital role in early detection of injury which ultimately results in rapid recovery of the patient. From the studies it has been proven that MRI being a most advanced and non-invasive medical imaging modality is excellent modality of choice for early and precise diagnosis of ligamentous injuries of the body including ACL, PCL tears of the knee joint 


\section{References}

1. Yaqoob J, Alam MS, Khalid N. Diagnostic accuracy of Magnetic Resonance Imaging in assessment of Meniscal and ACL tear: Correlation with arthroscopy. Pakistan journal of medical sciences. 2015;31(2):263.

2. Fatima R, Sadiq A, Sadiq S, Nazir M, Shazhad H, Mehmood A, et al. Frequency of Different Ligament Tears in Knee Injury On Magnetic Resonance Imaging. Methods. 2020;71.

3. El Adalany MA, Sakarana AAS, Fattah SA. The role of magnetic resonance imaging (MRI) in assessment of ACL graft Failure. The Egyptian Journal of Radiology and Nuclear Medicine. 2017;48(4):961-9.

4. Chang PD, Wong TT, Rasiej MJ. Deep learning for detection of complete anterior cruciate ligament tear. Journal of digital imaging. 2019;32(6):980-6.

5. Siegel L, Vandenakker-Albanese C, Siegel D. Anterior cruciate ligament injuries: anatomy, physiology, biomechanics, and management. Clinical Journal of Sport Medicine. 2012;22(4):349-55.

6. Guenoun D, Le Corroller T, Amous Z, Pauly V, Sbihi A, Champsaur P. The contribution of MRI to the diagnosis of traumatic tears of the anterior cruciate ligament. Diagnostic and Interventional imaging. 2012;93(5):331-41.

7. Sutton KM, Bullock JM. Anterior cruciate ligament rupture: differences between males and females. JAAOS-Journal of the American Academy of Orthopaedic Surgeons. 2013;21(1):41-50.

8. Cheung EC, Boguszewski DV, Joshi NB, Wang D, McAllister DR. Anatomic factors that may predispose female athletes to anterior cruciate ligament injury. Current sports medicine reports. 2015;14(5):36872.

9. Singh AP, Sharma S. Study of Various Knee Injury Findings in Magnetic Resonance Imaging (MRI).

10. Hooda A, Dhillon MS, Prabhakar S, Prakash M, John R, Kanwat H. MRI evaluation of anterolateral ligament tears in knee injury with anterior cruciate ligament rupture. Muscles, Ligaments \& Tendons Journal (MLTJ). 2018;8(1).

11. Kam C, Chee DW, Peh WC. Magnetic resonance imaging of cruciate ligament injuries of the knee. Canadian Association of Radiologists Journal. 2010;61(2):80-9.

12. Helito CP, Helito PVP, Costa HP, Bordalo-Rodrigues M, Pecora JR, Camanho GL, et al. MRI evaluation of the anterolateral ligament of the knee: assessment in routine 1.5-T scans. Skeletal radiology. 2014;43(10):1421-7.

13. Yoon JP, Yoo JH, Chang CB, Kim SJ, Choi JY, Yi JH, et al. Prediction of chronicity of anterior cruciate ligament tear using MRI findings. Clinics in orthopedic surgery. 2013;5(1):19-25.

14. Vaidya SV, Dhongade HS, Mahajan SM, Aneesh M. Evaluation of Anterior and Posterior Cruciate Ligament Injuries of the Knee on Magnetic Resonance Imaging: A Cross-Sectional Study. Int J Curr Res Rev. 2020;12:105-8.

15. Choi WR, Yang J-H, Jeong S-Y, Lee JK. MRI comparison of injury mechanism and anatomical factors between sexes in non-contact anterior cruciate ligament injuries. PLoS one. 2019;14(8):e0219586.

16. Gunaydin B, Sahin GG, Sari A, Kara A, Dincel YM, Cetin MU, et al. A new method for diagnosis of anterior cruciate ligament tear: MRI with maximum flexion of knee in the prone position: A case control study. International Journal of Surgery. 2019;68:142-7.

17. Prathap Kumar J, Kumar A, Venkatesh D. Healthy Gait: Review of Anatomy and Physiology of Knee Joint. Int J Cur Res Rev| Vol. 2020;12(06):1.

18. Ghazikhanian V, Beltran J, Nikac V, Feldman M, Bencardino JT. Tibial tunnel and pretibial cysts following ACL graft reconstruction: MR imaging diagnosis. Skeletal radiology. 2012;41(11):1375-9.

19. BArtHOLOMEEUSEN SCS, BEllEMANS J. High prevalence of anterolateral ligament abnormalities in magnetic resonance images of anterior cruciate ligament-injured knees. Acta Orthopædica Belgica. 2014;80:12014.

20. van der List JP, Mintz DN, DiFelice GS. The location of anterior cruciate ligament tears: a prevalence study using magnetic resonance imaging. Orthopaedic Journal of Sports Medicine. 2017;5(6):2325967117709966.

21. Bien N, Rajpurkar P, Ball RL, Irvin J, Park A, Jones E, et al. Deep-learning-assisted diagnosis for knee magnetic resonance imaging: development and retrospective validation of MRNet. PLoS medicine. 2018;15(11):e1002699.

22. Kunze KN, Rossi DM, White GM, Karhade AV, Deng J, Williams BT, et al. Diagnostic performance of artificial intelligence for detection of anterior cruciate ligament and meniscus tears: A systematic review.

Arthroscopy: The Journal of Arthroscopic \& Related Surgery. 2020.

23. Liu F, Guan B, Zhou Z, Samsonov A, Rosas H, Lian K, et al. Fully automated diagnosis of anterior cruciate ligament tears on knee MR images by using deep learning. Radiology: Artificial Intelligence.

2019;1(3):180091. 
24. Devitt BM, O’Sullivan R, Feller JA, Lash N, Porter TJ, Webster KE, et al. MRI is not reliable in diagnosing of concomitant anterolateral ligament and anterior cruciate ligament injuries of the knee. Knee Surgery, Sports Traumatology, Arthroscopy. 2017;25(4):1345-51.

25. Hartigan DE, Carroll KW, Kosarek FJ, Piasecki DP, Fleischli JF, D'Alessandro DF. Visibility of anterolateral ligament tears in anterior cruciate ligament-deficient knees with standard 1.5-Tesla magnetic resonance imaging. Arthroscopy: The Journal of Arthroscopic \& Related Surgery. 2016;32(10):2061-5.

\begin{tabular}{|c|c|c|c|c|c|}
\hline \multicolumn{6}{|c|}{ Gander } \\
\hline & & Frequency & Percent & Valid Percent & $\begin{array}{l}\text { Cumulative } \\
\text { Percent }\end{array}$ \\
\hline \multirow[t]{3}{*}{ Valid } & Female & 20 & 33.3 & 33.3 & 33.3 \\
\hline & Male & 40 & 66.7 & 66.7 & 100.0 \\
\hline & Total & 60 & 100.0 & 100.0 & \\
\hline
\end{tabular}

Number and Percentage of female and male patients in the study

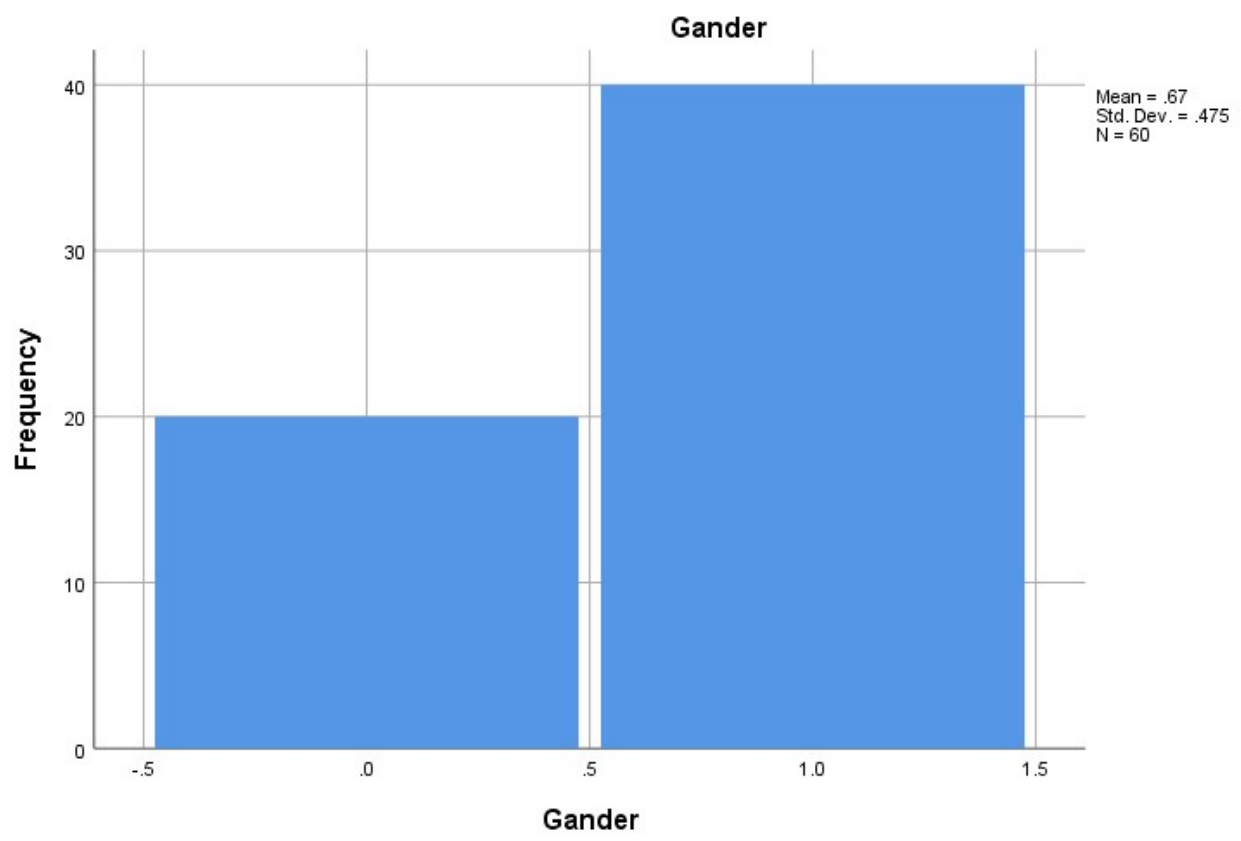




\begin{tabular}{|c|c|c|c|c|c|}
\hline \multicolumn{6}{|c|}{ Age(Years) } \\
\hline & & Frequency & Percent & Valid Percent & $\begin{array}{c}\text { Cumulative } \\
\text { Percent }\end{array}$ \\
\hline \multirow[t]{26}{*}{ Valid } & 18 & 2 & 3.3 & 3.3 & 3.3 \\
\hline & 19 & 1 & 1.7 & 1.7 & 5.0 \\
\hline & 20 & 1 & 1.7 & 1.7 & 6.7 \\
\hline & 24 & 1 & 1.7 & 1.7 & 8.3 \\
\hline & 25 & 1 & 1.7 & 1.7 & 10.0 \\
\hline & 26 & 10 & 16.7 & 16.7 & 26.7 \\
\hline & 28 & 2 & 3.3 & 3.3 & 30.0 \\
\hline & 29 & 5 & 8.3 & 8.3 & 38.3 \\
\hline & 30 & 5 & 8.3 & 8.3 & 46.7 \\
\hline & 32 & 4 & 6.7 & 6.7 & 53.3 \\
\hline & 33 & 1 & 1.7 & 1.7 & 55.0 \\
\hline & 34 & 1 & 1.7 & 1.7 & 56.7 \\
\hline & 35 & 3 & 5.0 & 5.0 & 61.7 \\
\hline & 36 & 3 & 5.0 & 5.0 & 66.7 \\
\hline & 38 & 2 & 3.3 & 3.3 & 70.0 \\
\hline & 40 & 4 & 6.7 & 6.7 & 76.7 \\
\hline & 44 & 2 & 3.3 & 3.3 & 80.0 \\
\hline & 45 & 1 & 1.7 & 1.7 & 81.7 \\
\hline & 47 & 1 & 1.7 & 1.7 & 83.3 \\
\hline & 49 & 2 & 3.3 & 3.3 & 86.7 \\
\hline & 50 & 1 & 1.7 & 1.7 & 88.3 \\
\hline & 51 & 2 & 3.3 & 3.3 & 91.7 \\
\hline & 55 & 1 & 1.7 & 1.7 & 93.3 \\
\hline & 61 & 1 & 1.7 & 1.7 & 95.0 \\
\hline & 62 & 3 & 5.0 & 5.0 & 100.0 \\
\hline & Total & 60 & 100.0 & 100.0 & \\
\hline
\end{tabular}

Occurrence of ACL with age 


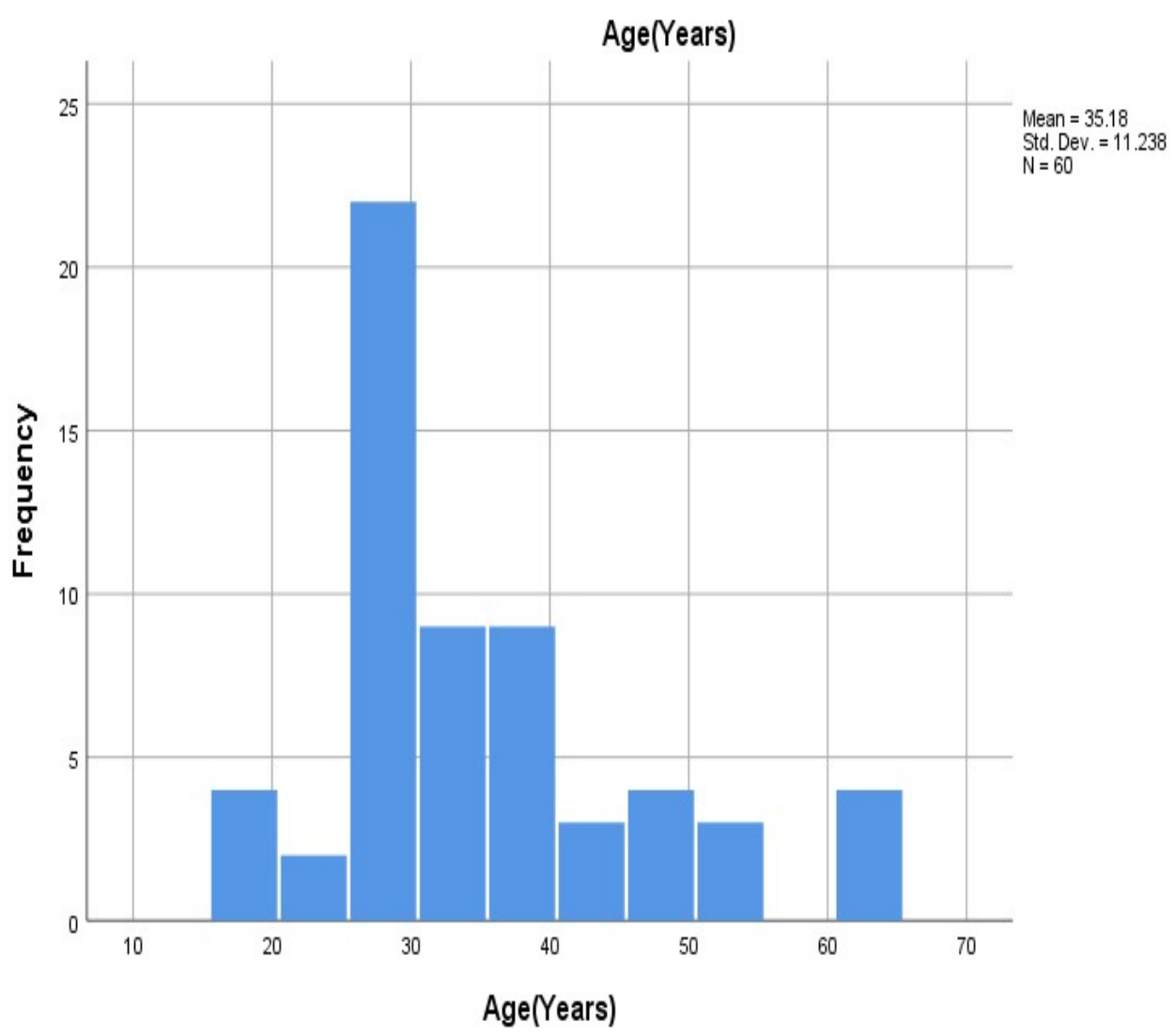

\begin{tabular}{|c|c|c|c|c|c|}
\hline \multicolumn{6}{|c|}{ Swelling } \\
\hline & & Frequency & Percent & Valid Percent & $\begin{array}{l}\text { Cumulative } \\
\text { Percent }\end{array}$ \\
\hline \multirow[t]{3}{*}{ Valid } & $\mathrm{NO}$ & 21 & 35.0 & 35.0 & 35.0 \\
\hline & YES & 39 & 65.0 & 65.0 & 100.0 \\
\hline & Total & 60 & 100.0 & 100.0 & \\
\hline
\end{tabular}

Number and percentage of patients presenting with swelling 


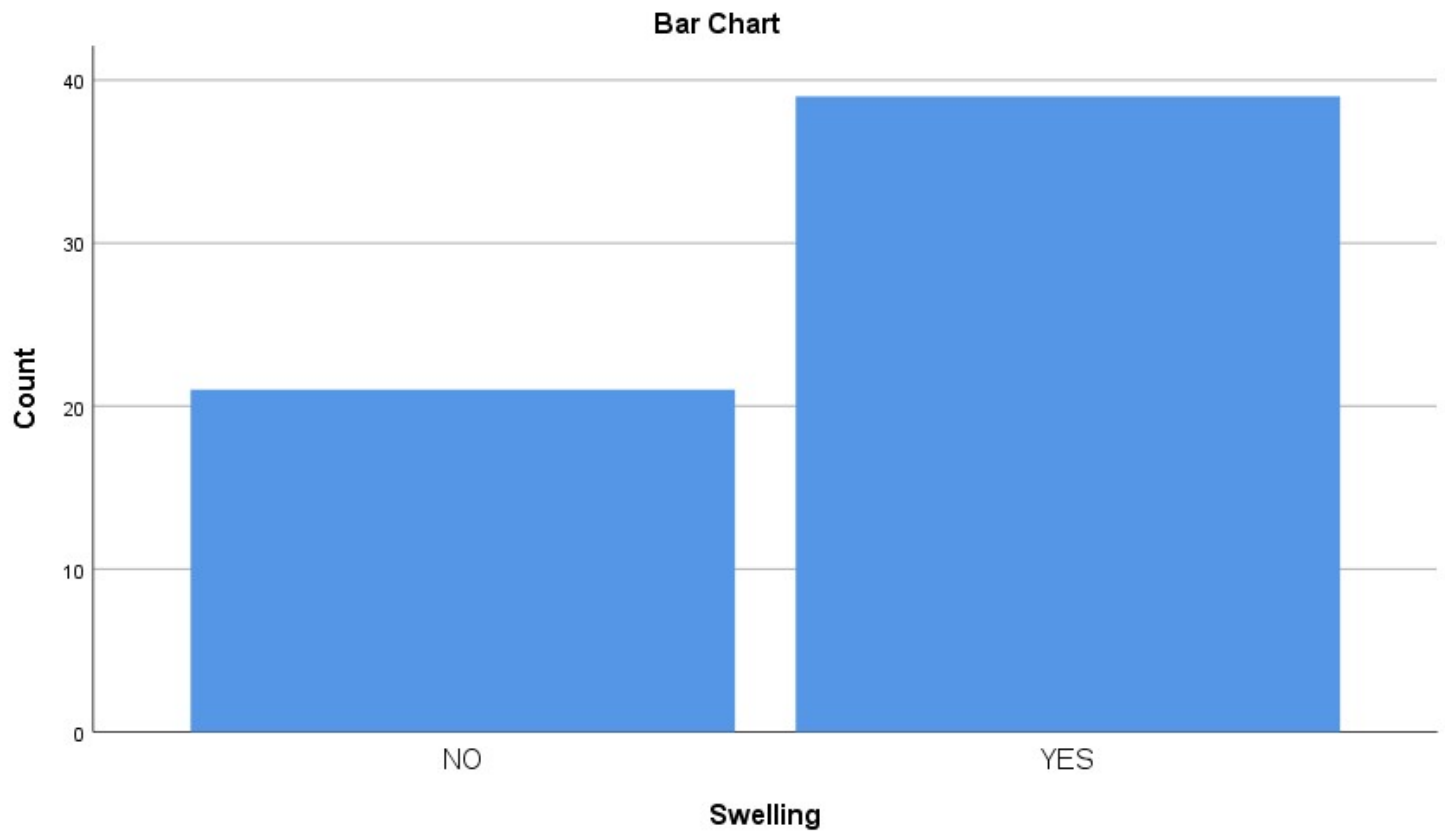

Pain during Movement

\begin{tabular}{|c|c|c|c|c|c|}
\hline & & Frequency & Percent & Valid Percent & $\begin{array}{c}\text { Cumulative } \\
\text { Percent }\end{array}$ \\
\hline Valid & YES & 60 & 100.0 & 100.0 & 100.0 \\
\hline
\end{tabular}

All the patients presented to the radiology department with suspected ACL tear Suffering from severe pain.

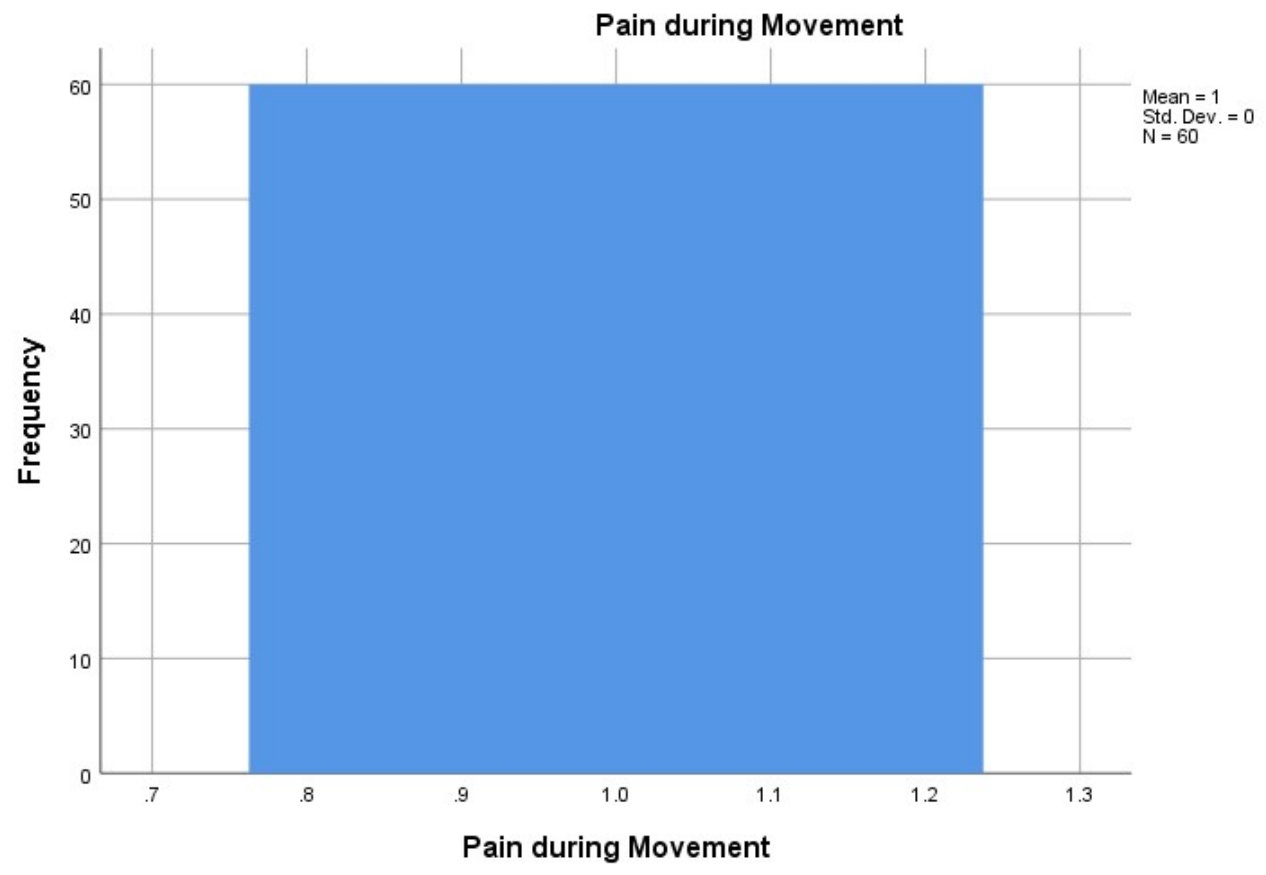




\begin{tabular}{c|cc|c|r|r}
\multicolumn{2}{c}{} & \multicolumn{2}{c}{ Diagnostic findings } & \multicolumn{2}{c}{$\begin{array}{c}\text { Cumulative } \\
\text { Percent }\end{array}$} \\
\hline \multirow{2}{*}{ Valid } & Frequency & Percent & Valid Percent & 25.0 \\
\cline { 2 - 6 } & Absent & 15 & 25.0 & 25.0 & 100.0 \\
\cline { 2 - 6 } & Present & 45 & 75.0 & 75.0 & \\
\cline { 2 - 6 } & Total & 60 & 100.0 & 100.0 & \\
\hline
\end{tabular}

Indicates the presence and absence of ACL tear in given sample

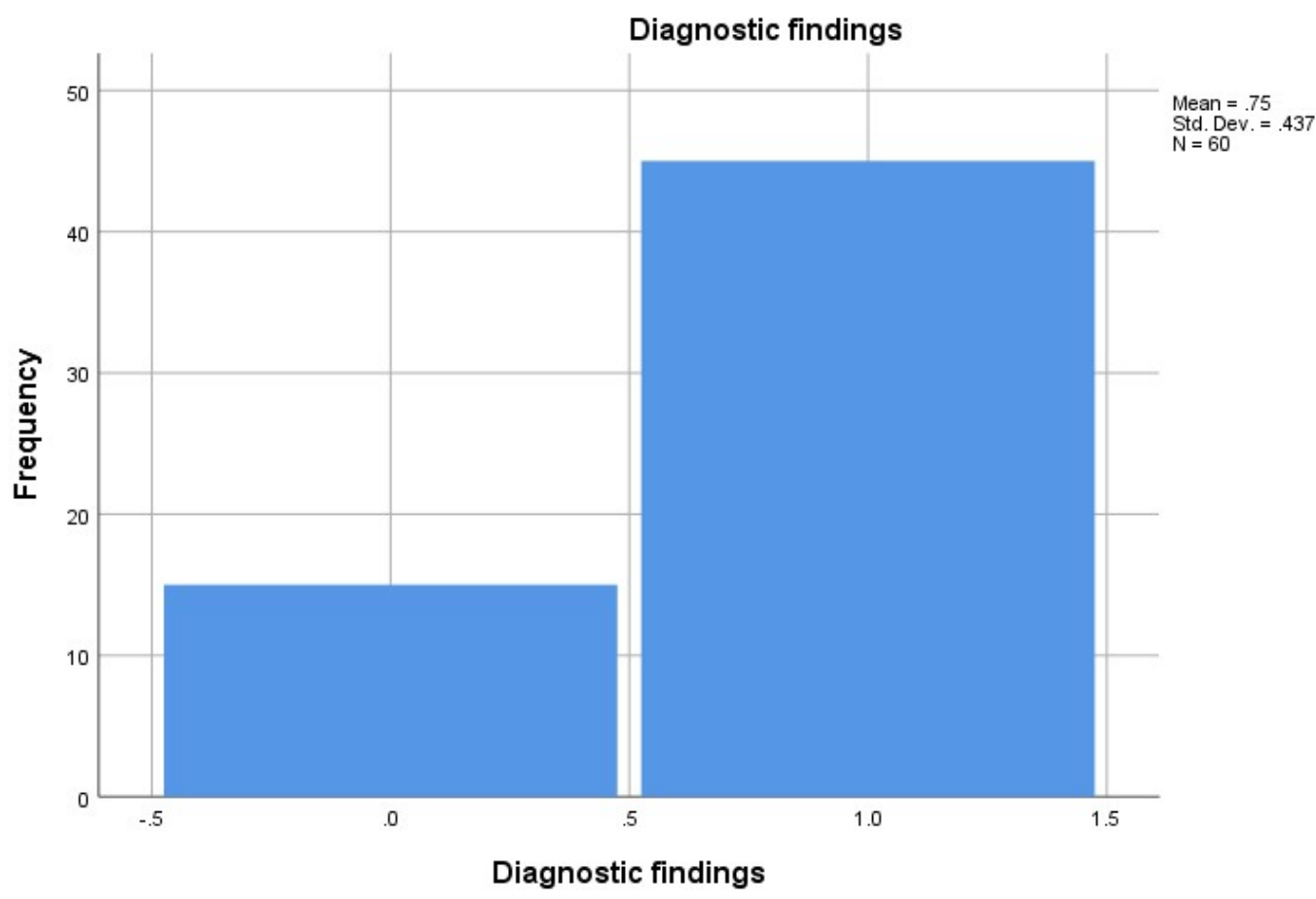

\begin{tabular}{lll|r|r|r}
\multicolumn{1}{c}{} & \multicolumn{2}{c}{ ACL Tears } & & \multicolumn{2}{c}{$\begin{array}{c}\text { Cumulative } \\
\text { Percent }\end{array}$} \\
\hline \multirow{3}{*}{ Valid } & Frequency & Percent & Valid Percent & 28.3 \\
\cline { 2 - 6 } & Partial Tear & 17 & 28.3 & 28.3 & 75 \\
\cline { 2 - 6 } & Complete Tear & 28 & 46.6 & 46.6 & 100.0 \\
\cline { 2 - 6 } & Intact & 15 & 28.3 & 28.3 & \\
\cline { 2 - 6 } & Total & 60 & 100.0 & 100.0 & \\
\hline
\end{tabular}



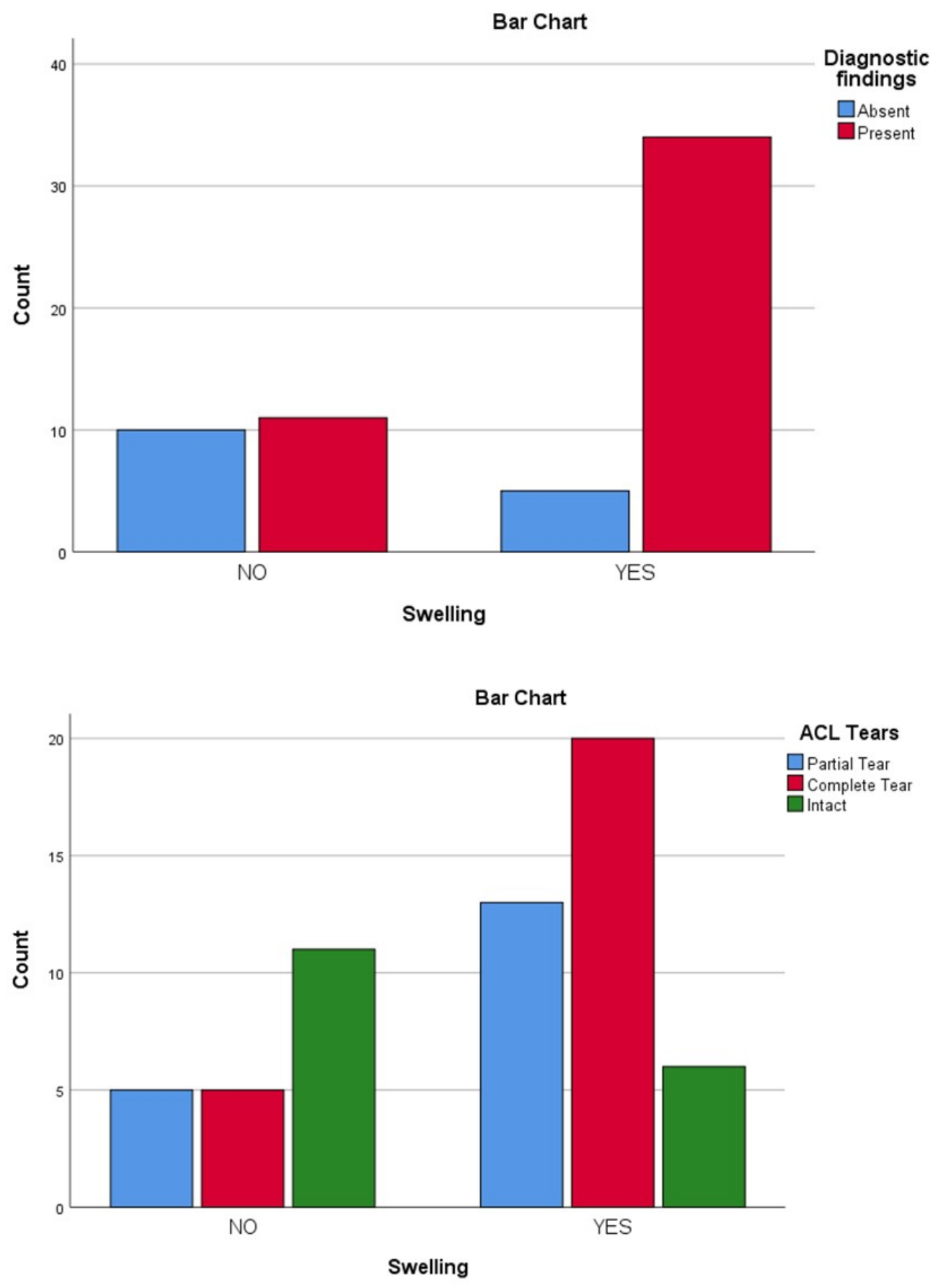